\title{
A SNP for hepatitis $C$ treatment failure
}

Genome-wide association results from the study by Tanaka et al. The dots with showed genome-wide significance. Image is reproduced, with permission, from Tanaka, Y. et al. (C) (2009) Macmillan Publishers Ltd. All rights reserved. arrows indicate SNPs that

Three new papers report a link between variants in the interleukin-28B (IL28B) gene and lack of response to treatment for hepatitis $\mathrm{C}$ virus (HCV) infection.

About $3 \%$ of the world's population is infected with HCV, which is the leading cause of liver cirrhosis and hepatocellular carcinoma in the United States. The current treatment fails in $40-50 \%$ of patients who have one of the major classes of HCV

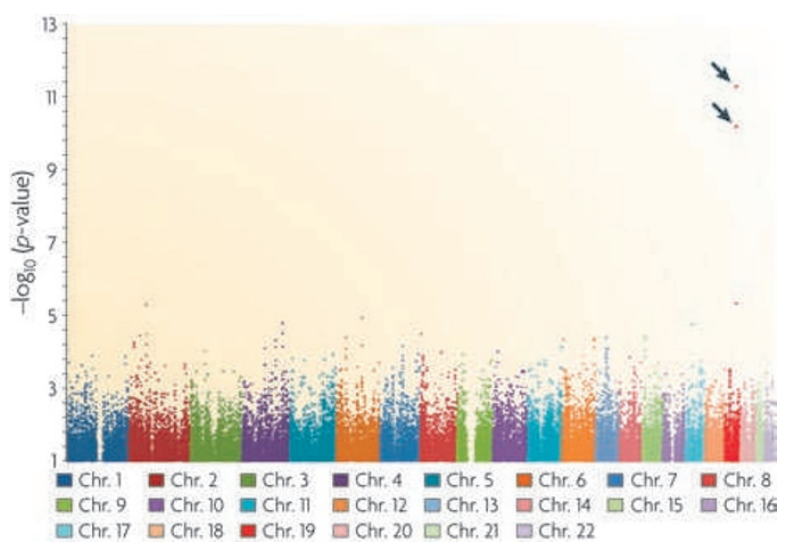

infection, and it can have serious side effects. To look at possible genetic reasons for the variation in response to treatment, the three groups performed genome-wide association studies. All three studies identified markers near the gene that encodes IL28B (also known as interferon- $\lambda 3$ (IFN $\lambda 3$ )), which, together with IL28A and IL29, is part of the type III interferon family. These cytokines are upregulated upon virus infection and have antiviral activity. IL29 is currently in Phase II trials as an anti-HCV treatment.

To identify the causal variants, $\mathrm{Ge}$ and colleagues sequenced IL $28 B$ in 96 individuals. They discovered two variants that were strongly associated with the marker they had originally identified. One variant was upstream of the translation start site and the other was in the coding region, although the effect of these variants remains unclear. Tanaka and colleagues and Suppiah and colleagues determined that in patients who have the marker that is associated with treatment failure, the levels of IL28 mRNA in the blood were significantly lower than in individuals who did not carry the marker, which suggests that the causal variants might affect the expression of this gene.

These markers may become part of a screen that identifies individuals who have high probabilities of treatment failure or success, and they are likely to become important stratifiers in trials of new compounds. Such tests could also help to refine and personalize treatment options for patients with chronic HCV infection.

Christiaan van Ooij, Chief Editor, Nature Reviews Microbiology

ORIGINAL RESEARCH PAPERS Ge, D. et al. Genetic variation in IL28B predicts hepatitis C treatment-induced viral clearance. Nature $\mathbf{4 6 1}$, 399-401 (2009) | Tanaka, Y. et al. Genome-wide association of IL28B with response to pegylated interferon- $\alpha$ and ribavirin therapy for chronic hepatitis C. Nature Genet. 41, 1105-1109 (2009)| Suppiah, V. et al. IL28B is associated with response to chronic hepatitis $C$ interferon- $\alpha$ and ribavirin therapy. Nature Genet. 41, 1100-1104 (2009) 